flux bound. These models have extremely rapidly rotating cores with the ratio $\lambda$ of centrifugal to gravitational forces being as high as $\frac{1}{4}$ to $\frac{1}{2}$.

But a serious flaw in the calculations has recently been pointed out by Monaghan (Mon. Not, R. astr. Soc., $169,13 \mathrm{P} ; 1974)$. The rapidly rotating models were computed under the assumption that $\lambda$ is very small, and approximations essentially equivalent to using $1+n \alpha \lambda$ for $(1+\alpha \lambda)^{n}$, where $\alpha$ is of order unity, were used. In the calculations of the neutrino flux $n$ is typically between 25 and 30 , and for such values, Monaghan proints out, the approximation leads to gross underestimates when $\lambda$ is as large as Demarque et al. and Roxburgh require, whatever the sign of $\alpha$. It seems, therefore, that even allowing for the errors arising from this approximation in the calculation of the mean energy generation rates, the quoted theoretical neutrino fluxes have been underestimated by at least a factor three.

A second criticism is raised in today's issue of Nature. Rood and Ulrich (page 366) have repeated the calculations of Demarque et al. and have argued that the lattcr authors made a mistake in their computation of the oblateness of their models. This is admitted by Demarque et al. on page 368. When the mistake is put right, the oblateness comes out to be greater than the measured value that $R$. H. Dicke reports. Roxburgh's model is not ruled out by Dicke's measurements, but it is contradicted by the recently announced results of the more careful investigations by $\mathrm{H}$. A. Hill and his collaborators, which imply an almost spherical Sun consistent only with a rotation of the interior hardly faster than that at the surface.

Rocd and Ulrich go on to investigate the combined effect of rapid rotation and a postulated transient convective mixing triggered by shear turbulence. Of course this raises the obvious difficulty of justifying why the Sun is in a unique peculiar transient state at the present time; but in any case the models presented today fail to yield sufficiently low neutrino fluxes combined with an adequately small oblateness, even though they are based on

\title{
Raindrops in the laboratory
}

from our Chemical Physics Correspondent

Drops of water, and eventually rain, grow from small droplets as they fall under gravity through slightly supersaturated air. Such a system is difficult to study in the laboratory as the droplets whose growth is of interest do not remain long in the field of view of any microscope and, unlike snowflakes, the drops can not reasonably be collected and studied at leisure.

Gollub, Chabay and Flygare ( $J$. chem. Phys., 61, 2139; 1974) have applied the special properties of the argon ion laser to this problem with great success. Basically the droplets scatter the laser light and, because of their falling motion, they impart a Dopper shift to the light frequency of the order of $500 \mathrm{~Hz}$. The exact shift will depend on the velocity of fall of the droplet through the viscous air and hence on the size of the droplet which can therefore be deduced from the Doppler shift. The shift can be accurately measured by beating the scattered light with light, unshifted in frequency, scattered from the stationary walls. The effective scattering volume is only $0.02 \mathrm{~cm}^{3}$ and is well defined in location so that the droplet size can be measured as a function of position in the sample chamber. This is $3.5 \mathrm{~cm}$ high internally and $10 \mathrm{~cm}$ in diameter so that control of temperature, of air purity and of super- saturation are straightforward to apply. Clearly different conditions are possible but in the experiments reported at $7^{\circ} \mathrm{C}$ and a degree of supersaturation of the order of $4 \%$, the mean droplet radius is about $3 \mu \mathrm{m}$ towards the top of the chamber and increases to about $7 \mu \mathrm{m}$ as the particle falls to the bottom. At any specific point the distribution of drop sizes about the mean for that point is remarkably narrow, for example, $\pm 0.2 \mu \mathrm{m}$ in $4 \mu \mathrm{m}$ about $2 \mathrm{~cm}$ below the point at which nucleation occurs.

Confirmatory evidence on the particle size measurement is obtained from the scattering intensity. The growth of the droplets as a function of the distance they have fallen provides information about the growth mechanism; the results support a reasonably high thermal accommodation coefficient and even the value unity is not excluded. The condensation coefficient seems to be less than unity but doubtless further work will improve the details of the theory of drop growth. Though the results from these first experiments are important, probably even more important is the introduction of a new, informative and fairly simple and inexpensive technique which will enable the formation of water droplets and of rain to be extensively studied in the future. the small $\lambda$ approximation and are subject to Monaghan's criticism. Rood and Ulrich conclude that rapid rotation is not a clue to the solution of the neutrino puzzle. They are probably right.

D. O. GovGH

\section{Inbreeding to preserve diversity}

from our Animal Ecology Correspondent IN the Uganda kob antelope there exists a system of social behaviour designed to prevent clandestine matings. Individual males fight for and guard small territories of between 10 and $20 \mathrm{~m}$ in diameter. A cluster of 30 to 40 such territories is called a lek. In the Torro Game Reserve in Western Uganda there are 18 such leks in an area of $400 \mathrm{~km}^{2}$. Courtship and mating take place within the territories am:d a complex and ritualised dressage which seems to be designed as if to publicise the fact that a mating has taken place. The details of the lek behaviour are well known (Buechner, Science, 133, 698; 1961; Proc 16th int. Congr. Zool, 3, 59; 1963), but what is not well known is why clandestine matings should be frowned upon.

Buechner and Roth (Am. Zool., 14, 145 ; 1974) have investigated the possibility that the total population is divided into socially defined breeding demes, each of which occupies a lek. Most leks persist for long periods in the same place-of the 15 major leks serving the Torro Reserve now, 10 are in traditional locations which can be dated back at least to 1959 . Evidence that animals breed only within a lek comes from tagging data and from translocation experiments. Most territorial males are found within $500 \mathrm{~m}$ of their home lek 90 to $100 \%$ of the time. Females are less attached to the site but all known individuals returned to the home lek for breeding. Until they reach $1 \frac{1}{2}$ years when they settle down in a lek, fawns seem to have little attachment to any particular site but on no occasion did an adult male translocated to a foreign lek succeed in establishing itself.

Morphological data, such as ear, tail and hind leg length, provide further evidence of inbreeding. There was significant variation between leks in males' hind leg and ear length, and females' body and tail length. Yet more evidence came from the animals' altruistic behaviour which, according to Hamilton (Man and Beast: Comparative Social Behaviour, edit. by J. F. Eisenberg and W. S. Dillon, 59 Smithsonian Institute Press, Washington; 1971) is directly related to the degree of kinship within a population. 